\title{
Acceso a la información pública y gestión documental: la experiencia uruguaya
}

Access to public information and document management: the Uruguayan experience

\author{
Alejandra Villar \\ Universidad de la República. Facultad de Información y \\ Comunicación, Instituto de Información, Uruguay \\ alejandra.villar@fic.edu.uy \\ (DD https://orcid.org/0000-0003-4379-7251
}

\begin{abstract}
Resumen:
El derecho de acceso de las personas a la información pública obliga a revisar el papel que realmente deben cumplir los archivos en las organizaciones y a ir corrigiendo las distorsiones y las carencias que se vayan presentando. Para fortalecer cualquier ley de derecho de acceso a la información pública es necesario contar con herramientas eficientes y eficaces en la creación, el mantenimiento, el uso y la disposición final de los documentos que las instituciones públicas generan y conservan. En el presente trabajo se realiza una reflexión sobre el derecho de acceso a la información pública y su vínculo con la gestión de documentos y archivos, así como el camino transitado en Uruguay a través de las acciones de la Unidad de Acceso a la Información Pública, en su vínculo con otros actores nacionales e internacionales.
\end{abstract}

Palabras ClaVe: Gestión de documentos y archivos, Archivología, Acceso a la información pública, Uruguay.

\section{ABSTRACT:}

The right of access of people to public information obliges us to review the role that archives should really play in Organizations and to correct any distortions and deficiencies that arise. To strengthen any law on the right of access to public information it requires efficient and effective tools in the creation, maintenance, use and final disposal of the documents that public institutions generate and preserve. This article reflects on the right of access to public information and its connection with document and archive management, as well as the progress in Uruguay through the Public Information Access Unit's actions in its relation with other national and international actors.

KEYWORDS: Records and archives management, Archival science, Access to public information, Uruguay.

\section{INTRODUCCIÓN}

En el presente trabajo se desarrolla un acercamiento a la realidad del ejercicio del derecho de acceso a la información pública en su vínculo con la gestión documental, en el contexto del país: la República Oriental del Uruguay.

Se aborda en primera instancia, el concepto de derecho de acceso a la información para que, al tiempo de analizar su surgimiento en el país, revisar su evolución en la región.

Luego, se muestra la importancia de la relación del derecho de acceso a la información pública con la gestión de documentos y administración archivos, haciendo hincapié en el objetivo de este trabajo, que es mostrar la experiencia uruguaya, así como el trabajo realizado desde la Unidad de Acceso a la Información Pública (UAIP) en ese sentido.

Cuando se hace referencia a la palabra acceso, el Diccionario de la lengua española (RAE, 2014) aporta diferentes significados, tales como "acción de llegar o acercarse", "entrada o paso" o "entrada al trato o comunicación con alguien". Mientras que, cuando se alude al término como un derecho humano, las acepciones pueden ser múltiples: el derecho de acceso a la salud, el derecho a la cultura, el derecho a la justicia, al trabajo, a la energía, entre otros. 
El derecho de acceso a la salud fue considerado por la Organización Mundial de la Salud (OMS, 2015) como un derecho humano, al igual que la alimentación, la vivienda, el trabajo, la educación, la no discriminación, entre otros. Así como también, el derecho de acceso a la cultura, como generador de espacios en los que se construye democracia y como herramienta para transversalizar temas que se refieren al ejercicio de otros reconocidos en 1948 por la Organización de las Naciones Unidas (ONU) en el artículo 27 de su Declaración Universal de los Derechos Humanos (ONU, 1948): "toda persona tiene derecho a tomar parte libremente en la vida cultural de la comunidad, a gozar de las artes y a participar en el progreso científico y en los beneficios que de él resulten".

Dentro de un sistema democrático, uno de los derechos fundamentales es que haya justicia, lo que en un Estado de derecho se vincula con los principios de igualdad, vida y dignidad humana. Además se destaca el derecho de acceso universal a la energía como esencial para el desarrollo de las personas y para la lucha contra la pobreza. De este modo se garantiza a la población una energía asequible, segura, sostenible y moderna para todas las personas, tal como indica el Objetivo de Desarrollo Sostenible 7 (PNUD, 2015) del Programa de las Naciones Unidas para el Desarrollo, es un objetivo incuestionable.

En el mundo del derecho, el término acceso abre un gran abanico de posibilidades y se refiere, en muchos casos, a diversos conceptos que a veces no tienen relación entre sí.

\section{El DERECHO DE ACCESO A LA INFORMACIÓN}

El derecho de acceso a la información pública tiene como antecedente la Ley de Libertad de Prensa de Suecia del año 1766, en donde quedaron explícitos los principios de libre circulación y acceso de los documentos oficiales y también la prohibición de la censura previa. Se podría decir que ese 2 de diciembre de 1766 se publicó la primera ley de libertad de información en el mundo.

Posteriormente, en 1789 la Asamblea Nacional Constituyente francesa adoptó la Declaración de los Derechos del Hombre y del Ciudadano como primer paso para luego escribir la Constitución de la República, en cuyo artículo 10 se señala que "Nadie debe ser incomodado por sus opiniones, inclusive religiosas, siempre y cuando su manifestación no perturbe el orden público establecido por la Ley" (Francia, Asamblea Nacional Constituyente, 1789). El concepto de derecho a la información también está presente en la Declaración Universal de los Derechos Humanos, que en su artículo 19 expresa:

Todo individuo tiene derecho a la libertad de opinión y de expresión; este derecho incluye el de no ser molestado a causa de sus opiniones, el de investigar y recibir informaciones y opiniones, y el de difundirlas, sin limitación de fronteras, por cualquier medio de expresión (ONU, 1948).

En América Latina, se encuentra conceptualizado en la Convención Americana sobre Derechos Humanos, más conocida como Pacto de San José de Costa Rica (Conferencia Especializada Interamericana de Derechos Humanos, 1969), en donde se consagra la libertad de buscar, recibir y difundir información e ideas de toda índole, sin consideración de fronteras, ya sea de manera oral, por escrito, en forma impresa o artística, o por cualquier otro procedimiento (artículo 13, inciso 1, "Libertad de pensamiento y expresión") (OEA, 1969).

Una forma moderna del tradicional derecho a la información corresponde al concepto de acceso a la información pública, en el cual lo importante es acortar las distancias entre las personas y el Estado (Lavalle Cobo, 2009) y en donde los conceptos de información pública y transparencia cobran destacada relevancia.

En la Ley Modelo de Acceso a la Información Administrativa, desarrollada por la Secretaría General de la Organización de los Estados Americanos (OEA) a través del Departamento de Cooperación Jurídica de la Secretaría de Asuntos Jurídicos en el año 2010, el concepto de derecho de acceso a la información está ampliamente desarrollado:

Toda persona tiene derecho a solicitar y a recibir información completa, veraz, adecuada y oportuna, de cualquier órgano perteneciente a la administración central, descentralizada de entes autárquicos, empresas y sociedades del Estado, sociedades 
anónimas con participación estatal mayoritaria, empresas e instituciones autónomas, órganos desconcentrados de la administración, y en todas aquellas otras organizaciones empresariales donde el Estado tenga participación en el capital o en la formación de decisiones societarias; así como del Poder Legislativo y Judicial, salvo disposición en contrario, en cuanto a su actividad administrativa. Este derecho de información también comprende el derecho a acceder a las informaciones contenidas en actas y expedientes de la Administración Pública, así como a estar informada periódicamente, cuando lo requiera, de las actividades que desarrollan entidades y personas que cumplen funciones públicas, siempre y cuando este acceso no lesione un interés público preponderante o el derecho a la privacidad e intimidad de un tercero. Este derecho también incorpora la posibilidad de formular consultas sobre las competencias y atribuciones de las entidades y funcionarios públicos que en ella laboran y a obtener copia de los documentos que recopilen información sobre sus actividades en la ejecución de las competencias a su cargo (OEA, 2010, p. 1).

En este punto, es importante destacar que, si bien muchas veces los conceptos de derecho de acceso a la información pública y de transparencia se usan como sinónimos, seguramente por su vínculo estrecho, no representan lo mismo.

Como enuncia el profesor Carlos Delpiazzo (2013), además del principio de juridicidad como pilar de un Estado de derecho, existen tres principios generales que importan a la accesibilidad de la información que está en poder de los organismos públicos:

- El principio de publicidad de los actos de gobierno, que, tal como expresa el profesor Felipe Rotondo Tornaría (1982), deriva de la forma republicana de gobierno.

- El principio de transparencia, que supone algo más que la publicidad, pues “... la publicidad implica mostrar, pero la transparencia implica algo más que mostrar, implica dejar ver; simplemente que el actuar de la Administración se deje ver como a través de un cristal" (Delpiazzo, 2003). La transparencia genera confianza y garantiza el derecho a estar informado, por lo cual se vuelve una herramienta fundamental para conocer la gestión pública y evaluar sus resultados. La Administración no solamente debe servir, sino mostrar cómo sirve, lo que le exige hacer disponible la información que produce y conserva, así como permitir la participación en sus actos y el control de estos.

- El principio de participación, de acuerdo con el cual para que exista accesibilidad real corresponde que las personas sean informadas y consultadas en los asuntos que les conciernen; quien participa en los asuntos públicos debe, necesariamente, estar previamente informado sobre esos asuntos.

\section{La Constitución de la República}

Como señala Mendel (2009, p. 25): "la inclusión del derecho de acceso a la información entre los derechos y libertades constitucionalmente garantizados es una indicación clara de su condición como derecho humano fundamental". Si bien en la Constitución de la República Oriental del Uruguay no figura el derecho de acceso a la información pública como tal, el artículo 29 garantiza el derecho a la libertad de expresión, sujeto a las restricciones que pueda determinar la ley:

Es enteramente libre en toda materia la comunicación de pensamientos por palabras, escritos privados o publicados en la prensa, o por cualquier otra forma de divulgación, sin necesidad de previa censura; quedando responsable el autor y, en su caso, el impresor o emisor, con arreglo a la ley por los abusos que cometieren.

Por su parte, el artículo 72 es una cláusula abierta que vincula otros derechos con un gobierno democrático y republicano, el artículo 82 protege la naturaleza republicana del gobierno, y el artículo 332 avala la suscripción de instrumentos internacionales que refieren al derecho de acceso a la información pública, como la Declaración Universal de los Derechos Humanos y el Pacto de San José de Costa Rica, al indicar:

Los preceptos de la presente Constitución que reconocen derechos a los individuos, así como los que atribuyen facultades e imponen deberes a las autoridades públicas, no dejarán de aplicarse por falta de la reglamentación respectiva, sino que ésta será suplida, recurriendo a los fundamentos de leyes análogas, a los principios generales de derecho y a las doctrinas generalmente admitidas. 
Con base en estos artículos, la justicia uruguaya ha sostenido la constitucionalidad de la actual Ley de Derecho de Acceso a la Información Pública en reiteradas oportunidades, como por ejemplo en la sentencias de la Suprema Corte de Justicia 185/013, del 13 de marzo de 2013; en la sentencia de la Corporación 253/99, que estableció que "el derecho de acceso a la información pública es el medio que permite el ejercicio del control y el conocimiento de la actuación de los representantes del pueblo en la gestión u obtención del bien común”. Así como también en la sentencia 802/2018, en la que la Suprema Corte de Justicia consideró que, de acuerdo con la Ley 18.381, una vez que se incumple con un pedido de información pública en el plazo legalmente estipulado, el Estado tiene el deber de proporcionar la información, con lo que el solicitante tiene derecho de requerir judicialmente la entrega de la información mediante la promoción de un proceso jurisdiccional en el cual se resolverá sobre la pertinencia de su pedido. Según la Corporación, toda entidad pública debería tener presente que está vigente una ley que le impone el deber de "prever la adecuada organización, sistematización y disponibilidad de la información en su poder, asegurando un amplio y fácil acceso a los interesados". Por lo tanto, el eventual incumplimiento de esta obligación no debería tener como consecuencia la lesión o la frustración del derecho de quien solicitó la información pública.

También, es posible destacar otros fallos de la justicia en los que se realiza una importante defensa del derecho a acceder a la información pública. Por ejemplo, la sentencia 144/2011, dictada por el Juzgado Letrado de Primera Instancia en lo Contencioso Administrativo de Primer Turno, en autos caratulados "Asociación de la Prensa Uruguaya C/ URSEC Unidad Reguladora de Servicios de Comunicaciones Amparo"; la sentencia 204/2013 del Tribunal de Apelaciones en lo Civil de Primer Turno, en el que se reclamaron datos a la Administración Nacional de Educación Pública (ANEP) sobre el funcionamiento del sistema educativo. Allí se fundó que las excepciones al derecho a la información son de interpretación estricta y comprenden aquellas que la ley define como secretas o exceptuadas; la sentencia 104/2017 del Tribunal de Apelaciones en lo Civil de Sexto turno; o, más recientemente, la sentencia 74/2019 del Tribunal de Apelaciones en lo Civil de Primer Turno, que señala:

No puede olvidarse que el derecho de acceder a la información pública y de informar públicamente es uno de los derechos fundamentales más importantes para un Estado republicano, democrático y de derecho, por lo que tiene rango constitucional (arts. 72 y 29).En la actualidad el derecho de todas las personas a una información “oportuna, veraz e imparcial” sin censuras ni ocultamientos viene adquiriendo particular relevancia y ha sido incorporado expresamente en textos constitucionales recientes (Brewer, Allan. La libre expresión y el derecho a la información en la Constitución Venezolana de 1999, en Anuario de Derecho Constitucional Latinoamericano, 2002, 267-276). "Por su parte, tiene dicho la Suprema Corte de Justicia que tanto el derecho a la información como la libertad de prensa son "derechos tan trascendentes que pueden ser ubicados en un plano superior al de otros derechos civiles pues de ello depende la estructura de las relaciones entre el poder y la libertad" (Sentencia 253 de 13/10/99). Como señala Muñoz Lorente - citado en ese fallo— su prevalencia deriva fundamentalmente del interés público, de la función que cumplen como contribuyentes a la formación de opinión pública libre, inherente a todo sistema democrático.

En suma, se podría decir, tal como lo expresó el exministro de la Suprema Corte de Justicia Ricardo Pérez Manrique, la Ley 18.381 de acceso a la información pública ha salvado su primer test de constitucionalidad (Uruguay. Poder Judicial, 2014). 
TABLA 1

Países de América del Sur que cuentan con una ley de derecho de acceso a la información pública.

\begin{tabular}{|c|c|c|c|}
\hline PAÍSES & TÍTULO DE LA LEY & $\begin{array}{c}\text { FECHA DE } \\
\text { PROMULGACIÓN }\end{array}$ & FUENTE DE INFORMACIÓN \\
\hline Argentina & $\begin{array}{l}\text { Ley de Derecho de } \\
\text { Acceso a la } \\
\text { Información Pública }\end{array}$ & $14 / 09 / 2016$ & $\begin{array}{l}\frac{\text { https://www.argentina.gob.ar/nor }}{\text { mativa/nacional/ley-27275- }} \\
\text { 265949/texto }\end{array}$ \\
\hline Brasil & $\begin{array}{l}\text { Ley General de } \\
\text { Acceso a la } \\
\text { Información Pública } \\
\text { de Brasil }\end{array}$ & $18 / 11 / 2011$ & $\begin{array}{l}\frac{\text { http://www.planalto.gov.br/ccivil } 0}{3 / \text { Ato2011- }} \\
\underline{\text { 2014/2011/Lei/L12527.htm }}\end{array}$ \\
\hline Bolivia & $\begin{array}{l}\text { Ley del Defensor del } \\
\text { Pueblo (1997) y } \\
\text { Decreto Supremo } \\
26186\end{array}$ & $17 / 5 / 2005$ & $\begin{array}{l}\text { https://www.oas.org/es/sla/ddi/doc } \\
\text { s/acceso_informacion_base_dc_l } \\
\text { eyes_pais_BO_2.pdf } \\
\text { http://www.oas.org/es/sla/ddi/docs } \\
\text { lacceso informacion base dc le } \\
\text { yes_pais_BO 6.pdf }\end{array}$ \\
\hline Chile & $\begin{array}{l}\text { Ley de Transparencia } \\
\text { de la Función Pública } \\
\text { y de Acceso a la } \\
\text { Información de la } \\
\text { Administración del } \\
\text { Estado }\end{array}$ & 11/08/2008 & $\begin{array}{l}\frac{\text { https://www.oas.org/es/sla/ddi/doc }}{\text { s/acceso_informacion_base_dc_I }} \\
\text { eyes pais } \mathrm{CH} \text { 2.pdf }\end{array}$ \\
\hline Colombia & $\begin{array}{l}\text { Ley de Transparencia } \\
\text { y del Derecho de } \\
\text { Acceso a la } \\
\text { Información Pública } \\
\text { Nacional }\end{array}$ & $6 / 03 / 2014$ & $\frac{\text { https://www.oas.org/es/sap/dgpe/ }}{\underline{\text { ACCESO/docs/Colombia2014.pdf }}}$ \\
\hline Ecuador & $\begin{array}{l}\text { Ley Orgánica de } \\
\text { Transparencia y } \\
\text { Acceso a la } \\
\text { Información Pública } \\
\text { de Ecuador }\end{array}$ & $18 / 05 / 2004$ & $\begin{array}{l}\frac{\mathrm{https} \text { //www.oas.org/es/sla/ddi/doc }}{\text { s/acceso informacion_base_dc_I }} \\
\text { eyes pais E 2.pdf }\end{array}$ \\
\hline Paraguay & $\begin{array}{l}\text { Ley del Libre Acceso } \\
\text { Ciudadano a la } \\
\text { Información Pública y } \\
\text { Transparencia } \\
\text { Gubemamental }\end{array}$ & $18 / 09 / 2014$ & $\frac{\text { https://www.oas.org/es/sap/dgpe/ }}{\text { ACCESO/docs/Paraguay2014.pdf }}$ \\
\hline Perú & $\begin{array}{l}\text { Ley de Transparencia } \\
\text { y Acceso a la } \\
\text { Información Pública y } \\
\text { su Reglamento }\end{array}$ & $13 / 07 / 2002$ & $\begin{array}{l}\text { https://www.peru.gob.pe/normas/d } \\
\text { ocs/ley 27806.pdf } \\
\text { https://www.oas.org/es/sla/ddi/doc } \\
\text { s/acceso_informacion_base_dc_I } \\
\text { eyes pais PE 2.pdf }\end{array}$ \\
\hline Uruguay & $\begin{array}{l}\text { Ley de } \text { Derecho de } \\
\text { Acceso a la } \\
\text { Información Pública } \\
\text { de Uruguay }\end{array}$ & $17 / 10 / 2008$ & $\frac{\text { https://www.impo.com.uy/bases/le }}{\text { ves/18381-2008 }}$ \\
\hline Venezuela & $\begin{array}{l}\text { No cuenta con una } \\
\text { ley de derecho de } \\
\text { acceso a la } \\
\text { información pública. } \\
\text { Si existe marco } \\
\text { normativo r sobre } \\
\text { acceso a la } \\
\text { información en poder } \\
\text { del sector público al } \\
2002 \text { (Mirador } \\
\text { Democrático). }\end{array}$ & Sin dato & $\begin{array}{l}\text { https://www.oas.org/es/sla/ddi/doc } \\
\text { s/acceso informacion base dc I } \\
\underline{\text { eyes pais VE 2.pdf }}\end{array}$ \\
\hline
\end{tabular}

Fuentes: OEA, Plataforma digital única del Estado peruano, portal oficial del Estado argentino, UAIP, Transparencia Venezuela, AC Mirador Democrático. 


\section{La Ley de Derecho de Acceso a la Información Pública en Uruguay}

Como en buena parte de los países de la región, en Uruguay, la promulgación de la Ley de Derecho de Acceso a la Información Pública es relativamente reciente, tal como se observa en la Tabla 1.

En particular Uruguay incluyó en su Agenda Digital 2008-2010 el derecho de acceso a la información pública, generando un marco normativo específico para ello y creando organismos estatales responsables del cumplimiento de la ley, como es el caso de la UAIP, órgano desconcentrado de la Agencia de Gobierno Electrónico.

La promoción, el análisis y la aprobación de la mencionada Ley uruguaya se realizó de forma casi simultánea con otras dos leyes íntimamente vinculadas a la gestión de la información en general: la Ley del Sistema Nacional de Archivos y la Ley de Protección de Datos Personales. Cabe destacar que esta experiencia de trabajo conjunto de las tres leyes no es una característica frecuente, al menos en la región (Ramos y Villar, 2013).

A lo anterior, se deben sumar las particularidades del equipo que trabajó en la Ley de Acceso a la Información Pública. El Grupo de Acceso a la Información Pública estuvo conformado por organizaciones de derechos humanos, como Amnistía Internacional-Sección Uruguay, el Instituto de Estudios Legales y Sociales del Uruguay, y Servicio Paz y Justicia Uruguay. También organizaciones de defensa de la libertad de expresión, como la Asociación de la Prensa del Uruguay y la Asociación Mundial de Radios Comunitarias y entidades que trabajan por la transparencia del Estado, como Uruguay Transparente y la Asociación Ciudadana por los Derechos Civiles. Además se incluyeron colectivos como Archiveros sin FronterasSección Uruguay, la Asociación Uruguaya de Archiveros, y la Escuela Universitaria de Bibliotecología y Ciencias afines, que trabajan en la gestión de los documentos y los archivos (Torres, 2014). Muchos de estos organismos conformaron asimismo el grupo de trabajo para la redacción y la propuesta de la Ley del Sistema Nacional de Archivos.

En ambos casos, la presencia y participación de los archivólogos en la redacción de las leyes ponen de manifiesto la importancia y lo necesario -imprescindible - del vínculo entre el acceso a la información pública y la gestión de documentos y la administración de archivos. Esta relación es evidente en las leyes cuando se menciona el deber del Estado de conservar y organizar el "Patrimonio Documental de la Nación y de los documentos de gestión como instrumentos de apoyo a la administración, a la cultura, al desarrollo científico, y como elementos de prueba, garantía e información” y la responsabilidad de crear y mantener los archivos profesionalmente organizados, como forma de garantizar la plenitud del ejercicio del acceso a la información.

La aprobación de la Ley de Acceso a la Información Pública puso a nuestro país en un lugar destacado en materia de transparencia, desde donde se continúa trabajando y obteniendo resultados positivos.

Recientemente, según el Índice de Percepción de la Corrupción 2020, elaborado por la ONG Transparencia Internacional, Uruguay es el país menos corrupto de la región, con lo que consolida su posición de liderazgo en la zona e iguala el índice anterior (Transparencia Internacional, 2020).

El documento destaca que Uruguay es percibido como el país más transparente de América Latina, con un total de 71 puntos sobre 100 posibles, con lo que mejoró su puntaje respecto de la edición 2018 del informe, cuando alcanzó 70 puntos sobre 100 posibles.

El estudio también indica que Uruguay ocupa el segundo puesto entre los 32 países de la región de las Américas, por detrás de Canadá (77 puntos) y por encima de Estados Unidos (67). 


\title{
5. ACCESO A LA INFORMACIÓN PÚBLICA Y GESTIÓN DE DOCUMENTOS Y ARCHIVOS
}

\author{
Toda ley de acceso a la información es tan buena como la calidad de los archivos a los que \\ proporciona acceso. Tales derechos son de poca utilidad si no se crean documentos confiables \\ en primer lugar, si estos no pueden encontrarse cuando se necesitan, o si los arreglos para su \\ eventual archivo o destrucción son inadecuados. \\ Draft UK code of practice on records management. ${ }^{1}$
}

El surgimiento de una nueva forma de entender la relación entre los ciudadanos y la administración se ha manifestado en el mundo con fuerza en los últimos años. Además de los procesos de transformación de la administración en la búsqueda de gobiernos eficaces y eficientes, se trabaja para acercar el accionar del gobierno a los ciudadanos y facilitar el derecho a participar, o sea, la posibilidad de que el ciudadano intervenga activamente en el proceso de la toma de decisiones, como otra forma de la transparencia gubernamental.

Eficacia, eficiencia y transparencia, son conceptos que están estrechamente relacionados con el concepto de Gobierno Abierto, entendido este como una forma de gobernar y llevar adelante la gestión pública basada en los pilares de transparencia; participación ciudadana y colaboración; y rendición de cuentas; y en donde la innovación tecnológica es un instrumento al servicio de estos fundamentos.

El gobierno abierto se centra e incluye a la ciudadanía en el proceso de toma de decisiones, así como en la co-creación e implementación de políticas públicas, para fortalecer la democracia, la eficiencia de los servicios públicos, prevenir y combatir la corrupción y lograr sociedades igualitarias e inclusivas. Tal como indica la Carta Iberoamericana de Gobierno Abierto, este no es un fin en sí mismo, sino "un medio para fortalecer la democracia, robustecer la institucionalidad pública y promover el mejoramiento de la calidad de vida de todas las personas" (CLAD, 2016).

Esta forma de entender la gobernanza democrática desde el gobierno abierto permite, de forma práctica, incorporar creatividad, ideas y voluntades en la búsqueda de soluciones a los problemas públicos considerados como relevantes dentro de una comunidad, a la vez que fortalece la legitimidad política de los Estados y de los regímenes democráticos.

Interesa particularmente en este trabajo, considerar el pilar de gobierno abierto de transparencia y acceso a la información pública, conceptos que deben guiar la relación entre los gobiernos y la sociedad civil, en el manejo de los asuntos públicos. La transparencia considerada en sus tres aspectos, el primer lugar, el derecho de las personas de acceder a la información que se encuentra en poder del Estado (transparencia pasiva). Luego se alude a la obligación de los gobiernos de poner a disposición de las personas, de forma proactiva, oportuna, completa, accesible, veraz y sencilla, aquella información que recoge de las actividades realizadas por la administración, la forma de uso de los recursos, así como los resultados obtenidos (transparencia activa). Finalmente, la liberación de información sobre temas específicos en poder del Estado, en formatos abiertos, como una manera de contribuir al logro de objetivos institucionales y fortalecer el proceso de toma de decisiones (transparencia focalizada).

Es evidente, entonces, que la transparencia y el acceso a la información pública - uno de los pilares más potentes del gobierno abierto - se nutre de una gestión documental que tiene en su fundamento la dotación de documentos, información y datos de calidad y auténticos. Es un vínculo más que necesario, imprescindible.

Para fortalecer cualquier ley de derecho de acceso a la información pública es necesario contar con herramientas eficientes y eficaces en la creación, el mantenimiento, el uso y la disposición final de los documentos que las instituciones públicas generan y conservan. Todo ello puede traducirse en la existencia de un sistema de gestión de documentos que garantice la identificación de todos los documentos que se crean, ingresan y conservan en las instituciones y que permite el acceso a la información en los plazos y las condiciones establecidas, evitando cualquier tipo de suspicacia sobre la transparencia de esa organización y la forma en que se toman las decisiones y se gestionan los recursos públicos. A propósito, recientemente, la Ley Modelo Interamericana sobre Acceso a la Información Pública 2.0 ha vuelto a marcar esa importante relación entre la gestión de documentos, los archivos y el acceso a la información. 
La nueva Ley Modelo 2.0 es el resultado del trabajo realizado para dar cumplimiento a la resolución de la Asamblea General de la OEA AG/RES. 2905 (XLVII-O/17), que solicitó al Departamento de Derecho Internacional que, en conjunto con los Estados miembros y la sociedad civil, se identificaran aquellos aspectos de la Ley Modelo Interamericana sobre Acceso a la Información Pública del año 2010 que requirieran actualización o ampliación. Entre los temas que fueron identificados como prioritarios a considerar en esta nueva versión está la gestión documental.

En ese sentido, y considerando que el tema es sumamente técnico, se resolvió dejarlo en manos de consultores expertos y adjuntar como anexo a la Ley Modelo 2.0 una propuesta de Ley Modelo de Gestión Documental y su Guía de Aplicación.

Es importante destacar varios artículos de la Ley Modelo 2.0 en los que se vuelve a poner de manifiesto que el cumplimiento de las obligaciones de transparencia exige un cambio en la manera en que se gestiona, almacena y recupera la información para contribuir al incremento de la transparencia pública, tal como se constata en la tabla 2:

TABLA 2

Artículos de la Ley Modelo 20 relacionados con la gestión de documentos

\begin{tabular}{|c|c|c|}
\hline $\begin{array}{l}\text { CAPITULO II. } \\
\text { MEDIDAS PARA } \\
\text { PROMOVER LA } \\
\text { APERTURA }\end{array}$ & Artículo 6 & $\begin{array}{l}\text { Dentro de las clases de información clave sujetas a difusión } \\
\text { proactiva se considera necesario incluir el cuadro de } \\
\text { clasificación archivística y el catálogo de disposición } \\
\text { documental o instrumentos análogos. }\end{array}$ \\
\hline \multirow{2}{*}{$\begin{array}{l}\text { CAPÍTULO III. } \\
\text { ACCE SO A LA } \\
\text { INFORMACIÓN } \\
\text { QUE OBRA EN } \\
\text { PODER DE LAS } \\
\text { AUTORIDADES } \\
\text { PÚBLICAS }\end{array}$} & Artículo 17 & $\begin{array}{l}\text { Sobre la forma de exhibir los documentos, se dispone que se } \\
\text { facilitará el acceso a los documentos originales, en } \\
\text { instalaciones adecuadas para tales propósitos. }\end{array}$ \\
\hline & Artículo 18 & $\begin{array}{l}\text { Sobre las obligaciones del oficial de información, quien, entre } \\
\text { otros deberes, tendrá que “a) promover dentro de la Autoridad } \\
\text { Pública las mejores prácticas en relación con el mantenimiento, } \\
\text { archivo y eliminación de Documentos". }\end{array}$ \\
\hline \multirow{2}{*}{$\begin{array}{l}\text { CAPÍTULO III. } \\
\text { ACCESO A LA } \\
\text { INFORMACIÓN } \\
\text { QUE OBRA EN } \\
\text { PODER DE LAS } \\
\text { AUTORIDADES } \\
\text { PÚBLICAS }\end{array}$} & Artículo 20 & $\begin{array}{l}\text { Relacionado con el mantenimiento de los documentos, se } \\
\text { establece que: "El/la [autoridad responsable de archivos] } \\
\text { deberá elaborar, en coordinación con el Órgano Garante, un } \\
\text { sistema de mantenimiento de Documentos que será vinculante } \\
\text { para toda Autoridad Pública". }\end{array}$ \\
\hline & Artículo 21 & $\begin{array}{l}\text { Se mencionan los esfuerzos y las acciones que la autoridad } \\
\text { respectiva deberá realizar ante el extravío de documentos. }\end{array}$ \\
\hline $\begin{array}{l}\text { CAPITULO IV. } \\
\text { REGGIMEN DE } \\
\text { EXCEPCIONES }\end{array}$ & Artículo 46 & $\begin{array}{l}\text { En los casos en que se declara que la información no existe, se } \\
\text { entiende que es necesario que esta declaración siempre sea } \\
\text { probada con un proceso de investigación en las diferentes } \\
\text { unidades organizativas de la Institución. Si se comprueba que } \\
\text { efectivamente fue eliminada y esta es de interés público, } \\
\text { deberá reconstruirse (literal 6). }\end{array}$ \\
\hline \multirow{3}{*}{$\begin{array}{l}\text { CAPÍTULO VI. } \\
\text { ÓRGANO } \\
\text { GARANTE }\end{array}$} & Artículo 63 & $\begin{array}{l}\text { Entre las "Facultades y atribuciones del Órgano Garante se } \\
\text { considera necesaria la coordinación con el Archivo General, de } \\
\text { forma de generar criterios para la clasificación y la } \\
\text { conservación de los documentos y la organización de los } \\
\text { archivos. }\end{array}$ \\
\hline & Artículo 65 & $\begin{array}{l}\text { Sobre los "Informes de los sujetos obligados" se establece que } \\
\text { en los informes que los sujetos obligados deben presentar ante } \\
\text { el órgano garante, entre otros aspectos, se deberá informar } \\
\text { sobre las actividades realizadas para implementar una } \\
\text { adecuada gestión documental". }\end{array}$ \\
\hline & Artículo 68 & $\begin{array}{l}\text { Por otra parte, se considera una infracción administrativa la } \\
\text { destrucción de documentos sin la debida autorización. }\end{array}$ \\
\hline
\end{tabular}

Fuente: elaboración propia basada en Ley Modelo Interamericana 2.0 sobre Acceso a la Información Pública. 
Queda claro entonces que es necesario que las leyes de transparencia contemplen la gestión documental, ya que la calidad, la fiabilidad y la accesibilidad a los documentos públicos son características que permiten el éxito de las iniciativas de acceso a la información. Pero también es imprescindible que las leyes de archivos se adapten al marco legal sobre transparencia y acceso a la información, coordinando y vinculando ambos sistemas. Esa coordinación debe verse, además, reflejada en el trabajo entre el órgano rector de las políticas archivísticas y el garante del acceso a la información pública, en una articulación interdisciplinaria e interinstitucional (Villar, Ramos, Piccini y Hernández, 2020).

\section{LA EXPERIENCIA URUGUAYA}

Desde el inicio de su trabajo en el año 2009, la UAIP ha participado en actividades relacionadas con la gestión de los documentos y los archivos, y se ha preocupado de incluirlas en su agenda. En los seminarios internacionales sobre acceso a la información, organizados desde hace ocho años, así como en el ciclo "Charlas de café", de la Unidad de Protección de Datos Personales, se han incluido paneles, talleres e instancias de intercambio sobre la gestión documental y su vínculo con el acceso a la información pública.

Por otra parte, desde el año 2012 se instituyó por el Decreto 80/012 del Poder Ejecutivo el Premio a la Transparencia, entregado a los sujetos obligados por la Ley 18.381, como un reconocimiento que realiza la UAIP a los organismos que se destaquen con las mejores experiencias y prácticas relacionadas con la transparencia y como forma de reforzar el compromiso del país con la Alianza para el Gobierno Abierto.

En las bases del Premio a la Transparencia, modificadas por el Decreto 179/013 del 11 de junio de 2013, se incluye el rubro temático "Archivos" con el objetivo de "fomentar las buenas prácticas tendientes a la conservación y disponibilidad de los activos de información". Para la evaluación de ese rubro se han tenido en cuenta los requisitos de la normativa del país, en particular lo dispuesto en la Ley 18.220 del 20 de diciembre de 2007, y sus decretos reglamentarios 355/2012 del 31 de octubre de 2012, y ampliatorio 70/2015 del 3 de febrero de 2015, valorando, entre otros aspectos, que se esté en proceso de constitución o que ya se encuentre establecida la Comisión de Evaluación Documental Institucional.

En el marco del relacionamiento internacional y desde la red de intercambio de la cual la UAIP forma parte desde abril de 2011 como miembro pleno, la Red de Transparencia y Acceso a la Información (RTA), ha participado activamente como contraparte en el grupo de trabajo que dio origen al Modelo de Gestión Documental de la Red (MGD_RTA).

En el año 2014, la RTA llevó adelante dos proyectos tendientes a otorgar mayor dinamismo y alcance a su trabajo, en conjunto con el programa de cooperación regional europeo EUROsociAL II, el Proyecto Modelo Regional de Gestión Documental y Archivos (Proyecto Archivos) y el Proyecto Modelo de Medición Internacional de Transparencia (Proyecto Indicadores).

Para los países miembros de la Red estaba claro que no alcanza con tener muy buenas leyes de acceso a la información pública si estas no son reforzadas con políticas y lineamientos de trabajo en materia de gestión de documentos y administración de archivos que acompañen, permitan y coadyuven con el pleno ejercicio del derecho de acceso a la información y la transparencia en la Administración.

Resultó evidente que no poder disponer de información puede convertirse en un impedimento para la gestión de las solicitudes, mientras que una administración inapropiada de archivos podría retardar la recopilación de la información solicitada. En ese sentido, el objetivo del MGD_RTA es "convertirse en soporte y apoyo para la gestión documental y de archivos de las distintas organizaciones y países miembros de la Red, así como garantizar la accesibilidad a los documentos" (RTA, 2014, p. 1).

La elaboración del modelo desde el punto de vista teórico se llevó a cabo en el año 2014, con el apoyo de profesionales archivólogos de la Subdirección General de los Archivos Estatales del Gobierno de España, como consultores de EUROsociAL. Además de la recopilación de información y de buenas prácticas internacionales y de la aplicación de encuestas a órganos rectores de archivos, entidades garantes de acceso a 
la información y a especialistas, que sirvieron para la elaboración del informe diagnóstico, se llevaron a cabo dos talleres, uno en Bogotá (Colombia), para la aprobación de los atributos del Modelo, y otro en Brasilia (Brasil), para la aprobación final.

En Brasilia, los representantes de los países que formaron parte del proyecto firmaron un acuerdo con las siguientes responsabilidades:

reafirmar el compromiso de fortalecer el Proyecto que se ha llevado adelante, en tanto representa una contribución a las comunidades de acceso a la información y archivos" y "animar, a sus instituciones y colaboradores a generar mecanismos que permitan una implementación del MGD y sus herramientas, a fin de potenciar el desarrollo de las distintas actividades efectuadas por la RTA (RTA, 2014, p. 2).

Con el objetivo de contribuir con la implementación del MGD en la realidad normativa y técnica de Uruguay, pero también con el propósito de honrar el compromiso asumido en la Declaración de Brasilia, la UAIP desarrolló un proyecto de "Guías técnico-metodológicas de gestión documental y administración de archivos". El mismo fue llevado adelante por un trabajo conjunto entre el equipo técnico y miembros del Consejo Ejecutivo de la UAIP, funcionarios profesionales de la Agencia de Gobierno Electrónico y la Sociedad de la Información y del Conocimiento, docentes de la Licenciatura en Archivología de la Facultad de Información y Comunicación de la Universidad de la República, Además contaron con el apoyo de la Subdirección General de Archivos Estatales de España, en el marco del Programa de Cooperación de la Unión Europea con América Latina EUROsociAL+.

Otro proyecto de la UAIP que también aborda la gestión documental es el Índice Nacional de Transparencia y Acceso a la Información. Se trata de un compromiso de la UAIP, en el marco del Cuarto Plan de Acción Nacional de Gobierno Abierto 2018-2020 de Uruguay de la Agencia de Gobierno Electrónico y Sociedad de la Información y del Conocimiento, y se enmarca además en las acciones que el país viene desplegando para el cumplimiento del Objetivo de Desarrollo del Milenio 16 de "promover sociedades justas, pacíficas e inclusivas" (AGESIC, 2018). Este compromiso contribuye a reducir la corrupción, promover la creación de instituciones eficaces y transparentes que rindan cuentas, garantizar el acceso público a la información y proteger las libertades fundamentales, de conformidad con las leyes nacionales y los acuerdos internacionales suscritos por el país.

La UAIP trabajó en una primera versión de la metodología del Índice, que fue elaborada por su Equipo de Fiscalización y validada por su Consejo Ejecutivo. Ese trabajo fue posteriormente abordado y mejorado colaborativamente en dos mesas de diálogo que se llevaron a cabo en septiembre del año 2019. En estas instancias se contó con la participación de representantes de diferentes organismos del Estado, la sociedad civil y la academia vinculados con la estrategia de gobierno abierto que lleva adelante el país.

El trabajo en estas mesas de diálogo se realizó mediante una dinámica de equipos para avanzar en el diseño y la construcción de los compromisos asumidos, agrupados según las siguientes temáticas: alcance de la norma, transparencia pasiva y activa, institucionalidad de la UAIP, índice nacional de transparencia y las dimensiones de transparencia pasiva y activa e institucional. Entre los resultados obtenidos en los subgrupos de trabajo, se destaca lo sugerido por la comisión de transparencia pasiva. Allí se propuso adoptar como obligatorias las normas de gestión documental y archivos, vinculando en una futura reforma de la Ley de Derecho de Acceso a la Información Pública, la normativa vigente en materia de gestión documental y archivos, e incorporar como punto de difusión obligatorio dentro de la transparencia activa la información sobre los fondos documentales y las tablas de plazo precaucional de documentos.

En cuanto al índice nacional de transparencia, si bien hay consenso en ponderar los pesos de las dimensiones considerando el orden transparencia pasiva, activa e institucional, se considera importante destacar en la dimensión institucional los procesos de gestión documental y administración de archivos abordados en todos los soportes, analógicos y digitales. Este índice se aplicó de manera piloto en una muestra de 25 organismos públicos sujetos obligados de la Ley de Derecho de Acceso a la Información Pública pertenecientes a todos los niveles administrativos de gobierno. 


\subsection{El proyecto de las "Guías técnico-metodológicas de gestión documental y administración de archivos"}

El objetivo general del proyecto fue el diseño y la elaboración de guías técnico-metodológicas de gestión documental y de archivos para el uso de personal administrativo no especializado, así como la participación en foros nacionales de intercambio y talleres organizados por la UAIP, con el fin de avanzar en el conocimiento y la aplicación del Modelo de Gestión Documental elaborado para la RTA.

Los objetivos específicos del proyecto fueron:

1. Trasladar experiencias internacionales exitosas en la gestión documental y la administración de archivos (física o electrónica) a la realidad nacional.

2. Implementar una estrategia de socialización entre los participantes en la elaboración de las guías sobre la estructura y los contenidos que debe recoger dicha herramienta.

3. Generar un documento de guías técnicas considerando algunos de los procesos de gestión documental: control intelectual, evaluación documental, control físico y servicios de archivo.

4. Presentar públicamente los productos, para su mayor difusión y conocimiento.

Se realizó el análisis de diferentes experiencias internacionales exitosas en la gestión documental y la administración de archivos, como el proyecto realizado en El Salvador en 2018 Lineamientos del proyecto de normalización de la gestión documental y la protección de datos de carácterpersonal de los expedientes clinicos. Así como tambiénla redacción en México en 2017 del Manual de procedimientos de gestión documental y archivos del INAI; el proyecto "madre" de la RTA (2014) Modelo de gestión de documentosy administración de archivos para la Red de Transparencia y Acceso a la Información y el proyecto de Perú (2017) denominado Modelo de gestión documental en el marco del Decreto Legislativo $N^{\circ} 1310$. Completa estas referencias modélicas la iniciativa de la OEA (2018) Propuesta de guia de aplicación de la Ley Modelo Interamericana de Gestión Documental, la cual figura como anexo de la Ley Modelo Interamericana de Acceso a la Información Pública 2.0.

Luego de esta revisión de iniciativas, se decidió incorporar al proyecto de la UAIP, la mención en la normativa nacional de las recomendaciones propuestas por el MGD_RTA, como la experiencia desarrollada por El Salvador. También se resolvió utilizar diagramas de flujo para explicitar y normalizar los procedimientos de gestión documental que se desarrollen, como el manual redactado por el Instituto Nacional de Transparencia, Acceso a la Información y Protección de Datos Personales en México. Además, el uso de ideas fuerza y de elementos que generen sentido de marca, como colores, cuadros-resumen y aspectos vinculados con la estructura de los documentos, tal como está en el MGD_RTA. Cabe agregar la consideración de la solidez normativa de la propuesta de la OEA y el compromiso de revisar a través de indicadores y el uso de gráficos que aporten claridad visual y conceptual a la herramienta, tal como hizo el proyecto ejecutado en Perú.

La estructura básica de estas guías contiene los siguientes aspectos:

- definición de los términos principales, para una mejor comprensión de la lectura posterior;

- detalle de los compromisos que exija cada línea de acción;

- visualización de contenidos mediante diagramas de flujo;

- referencia de la normativa del país que sustenta los compromisos;

- inclusión de indicadores, como la necesidad de posterior seguimiento;

- inclusión de recursos, como referencias de bibliografía nacional, líneas de investigación o recursos en línea. 
La decisión adoptada por el grupo de que tanto la estructura de los documentos, como las líneas de acción que contienen las guías técnicas, fueran concordantes con los que figuran en la Guía de Aplicación de la Ley Modelo Interamericana de Gestión Documental, significa para Uruguay estar en sintonía y cumplir con la propuesta normativa panamericana.

La Ley modelo Interamericana de Gestión Documental 2.0 tiene ocho capítulos, de los cuales cuatro fueron incorporados a las guías técnicas de Uruguay: "Identificación, clasificación y descripción de documentos", "Valoración, transferencia y eliminación de documentos", "Conservación de documentos y gestión de contingencias", "Difusión y servicios de atención a los usuarios". La inclusión del 50\% de los contenidos de la Guía de Aplicación de la Ley Modelo en las guías técnico - metodológicas de Uruguay, además de representar una buena práctica y un modelo a seguir, significa un claro ejemplo de que es posible llevar la propuesta panamericana a un caso particular a nivel nacional.

\subsection{El Índice Nacional de Transparencia y Acceso a la Información}

El compromiso asumido por la UAIP y el objetivo principal para el trabajo en el índice fue:

Crear el Índice Nacional de Transparencia y Acceso a la Información (INTAI) que mida el nivel de madurez de los sujetos obligados en el cumplimiento de las obligaciones establecidas en la por la Ley No. 18.381 del 17 de octubre de 2008 de Acceso a la Información Pública y sus decretos reglamentarios. Elaborar un instrumento de evaluación general que permite medir el cumplimiento de los diferentes aspectos relacionados a la transparencia de la información pública en cada sujeto obligado por la Ley (AGECIC, 2018, p. 43).

Para la recolección de la información que ayude a medir la existencia de políticas, lineamientos, procesos, procedimientos o buenas prácticas documentadas, dentro de cada organismo sujeto obligado de la Ley de Derecho de Acceso a la Información Pública, se elaboró un formulario, ${ }^{2}$ que fue validado por el Consejo Ejecutivo de la UAIP y que se aplicó entre los 25 organismos que fueron parte del piloto en el año 2019.

El índice tiene tres dimensiones con diferente ponderación sobre un total de 100\%: transparencia activa (35\%), transparencia pasiva (40\%) y dimensión institucional (25\%). Interesa destacar el contenido de este último punto, pues es en el que se aborda el tema de la gestión documental en su relación con el acceso a la información pública (UAIP, 2019a). Esta dimensión institucional "evalúa los instrumentos y procedimientos internos con los que cuenta cada organismo para producir, publicar y difundir información, a fin de generar visibilidad de sus actividades y gestión, logrando así mayor conocimiento, contacto y acercamiento con el ciudadano" (UAIP, 2020).

La distribución de la ponderación correspondiente a la dimensión institucional se subdividió de la siguiente forma, otorgando a la gestión documental y de archivo el $45 \%$ del total, como se ve en la Tabla 3. 
TABLA 3

INTAI, dimensión institucional.

\begin{tabular}{|c|c|c|c|}
\hline \multicolumn{4}{|c|}{ Dimensión institucional (25\%) } \\
\hline $\begin{array}{l}\text { Gestión documental } \\
\text { y de archivos }(\mathbf{4 5} \%)\end{array}$ & $\begin{array}{l}\text { Instrumentos y } \\
\text { procedimientos } \\
\text { internos }(30 \%)\end{array}$ & Datos abiertos $(15 \%)$ & Capacitación (10\%) \\
\hline \multirow{2}{*}{$\begin{array}{l}\text { GDA } 1 \text { a } 9: \\
\text { preguntas Ley } \\
\mathbf{1 8 . 2 2 0} \text { y su decreto } \\
\text { reglamentario } \\
\mathbf{3 5 5 / 2 0 1 2} \text { del } \\
\mathbf{3 1 / 1 0 / 0 1 2}(15 \% \\
\text { preguntas } 1.2,1.8 \text { y } \\
1.9 \text { cada una, } \\
\text { restantes preguntas, } \\
9,17 \% \text { cada una) }\end{array}$} & \multirow{2}{*}{\begin{tabular}{|l|} 
I1: Instrumentos y \\
procedimientos \\
intemos con los que \\
cuenta el organismo \\
para producir \\
información $(50 \%)$. \\
I2: Instrumentos y \\
procedimientos \\
intemos con los que \\
cuenta el organismo \\
para public ar y \\
difundir información \\
$(50 \%)$.
\end{tabular}} & $\begin{array}{l}\text { DA1: Disposición de } \\
\text { información general o } \\
\text { estadistica en formatos } \\
\text { reutilizables en el sitio } \\
\text { web }(40 \%) \text {. }\end{array}$ & \multirow{2}{*}{$\begin{array}{l}\text { C1: Capacitación a } \\
\text { funcionarios en } \\
\text { participación, } \\
\text { transparencia y } \\
\text { acceso a la } \\
\text { información }(100 \%) \text {. }\end{array}$} \\
\hline & & $\begin{array}{l}\text { DA2: Disposición de } \\
\text { información especifica de } \\
\text { transparencia activa en } \\
\text { formatos reutilizables en } \\
\text { el sitio web }(60 \%) \text {. }\end{array}$ & \\
\hline
\end{tabular}

Fuente: marco metodológico del UAIP (2019b).

De las 11 preguntas formuladas sobre gestión documental y de archivos, las preguntas 1 a 9 estuvieron relacionadas con normativa de carácter preceptiva para los sujetos obligados de la Ley del Sistema Nacional de Archivos y de Derecho de Acceso a la Información Pública, tal como puede apreciarse en la Tabla 4. De ellas, las preguntas 1.2, 1.8 y 1.9 refieren a obligaciones y procesos expresamente mencionados en las normas, por eso su valoración es de un 15\%, frente a las demás preguntas, que fueron ponderadas con un $9,17 \%$ cada una.

TABLA 4

Preguntas del cuestionario para referentes del INTAI y su vínculo con la normativa en materia de gestión de documentos y archivos.

\begin{tabular}{|c|c|c|c|}
\hline PREGUNTA & $\begin{array}{l}\text { LEY } \\
18.220\end{array}$ & \begin{tabular}{|c} 
DEC. \\
$355 / 201$ \\
2
\end{tabular} & $\begin{array}{c}\text { LEY } \\
18.381\end{array}$ \\
\hline $\begin{array}{l}\text { 1.1. i. Existe fondo(s) documental(es) v/o colecciones } \\
\text { identificado(s) v orqanizado(s) archivísticamente. cualquiera } \\
\text { sea el sooorte en donde esté contenida la información? }\end{array}$ & Art. 1 & Art. 6 & Art. 6 \\
\hline $\begin{array}{l}\text { 1.2. ¿Existen profesionales archivóloqos en los Archivos } \\
\text { Administrativos e Históricos. o en las Unidades de Gestión } \\
\text { Documental? }\end{array}$ & Art. 7 & $\begin{array}{l}\text { Arts. } 6 \\
\text { y } 13\end{array}$ & \\
\hline $\begin{array}{l}\text { 1.2. a. i.El carqo profesional se encuentra reqularizado o en } \\
\text { oroceso de creación en el escalafón orofesional? }\end{array}$ & Art. 7 & & \\
\hline $\begin{array}{l}\text { 1.3. ¿. Existen espacios físicos acondicionados para el } \\
\text { funcionamiento de los Archivos: con la loaística e } \\
\text { infraestructura necesaria para desarrollar los procesos } \\
\text { archivísticos? }\end{array}$ & Art. 1 & Art. 6 & \\
\hline $\begin{array}{l}\text { 1.4. Existe un espacio diaital acondicionado para el } \\
\text { funcionamiento de los Archivos diaitales. loaística e } \\
\text { infraestructura necesaria para los procesos archivísticos? }\end{array}$ & Art. 1 & $\begin{array}{c}\text { Arts. } 6 \\
\text { y } 8\end{array}$ & \\
\hline $\begin{array}{l}\text { 1.5. i.Existen Sistemas de sequridad de los documentos en } \\
\text { soporte físico (analóqico. papel) iunto con una qestión de los } \\
\text { riesaos definida e imblementada? }\end{array}$ & Art. 1 & Art. 6 & \\
\hline $\begin{array}{l}\text { 1.6. Existen Sistemas de sequridad de los documentos en } \\
\text { soporte diaital (respaldo. acceso, confidencialidad. } \\
\text { autenticidad v trazabilidad) iunto con qestión de los riesqos } \\
\text { definida e imblementada? }\end{array}$ & Art. 1 & $\begin{array}{l}\text { Arts. } 6 \\
\text { y } 8\end{array}$ & \\
\hline $\begin{array}{l}\text { 1.7. Z Están normalizados los procesos de reproducción de } \\
\text { los documentos (protocolos de microfilmación v de } \\
\text { diaitalización) v definidos los sistemas de preservación } \\
\text { diqital? }\end{array}$ & Art. 1 & \begin{tabular}{|l} 
Arts. 6 \\
8 y 9
\end{tabular} & \\
\hline $\begin{array}{l}\text { 1.8. i. Está constituida la Comisión de evaluación Documental } \\
\text { institucional establecida en la normativa? }\end{array}$ & Art. 1 & $\begin{array}{c}\text { Art. } 6 \text { lit. } \\
\mathrm{q}^{5}\end{array}$ & \\
\hline $\begin{array}{l}\text { 1.9. i.Están identificadas las series documentales v } \\
\text { elaboradas las Tablas de Plazos Precaucionales de } \\
\text { documentos? }\end{array}$ & Art. 1 & Art. $17^{6}$ & \\
\hline
\end{tabular}

Fuente: cuestionario a referentes del INTAI, leyes 18.220 y 18.381 y Decreto 355/2012.

El índice califica a cada institución en una escala de 0 a 100 puntos, con los siguientes niveles de cumplimiento: mayor a $80 \%$ se considera; entre $60 \%$ y $79 \%$ es mediano; y menos de $59 \%$ es bajo. Según los resultados generales del piloto realizado en el año 2019 por la UAIP, de los sujetos obligados participantes, es decir 20 organismos, "el $76 \%$ cumplen al menos parcialmente con las obligaciones de transparencia y acceso a la información pública establecidas en la normativa vigente” (UAIP, 2020).

Los resultados por dimensión destacan un mejor cumplimiento en las áreas de transparencia activa y pasiva, en las que la UAIP viene trabajando desde hace mucho tiempo y realizando fiscalizaciones frecuentes, frente a la dimensión institucional, en la que por primera vez se incorporan aspectos relacionados con la gestión de documentos y archivos. 
GRÁFICO 1

Resultados por dimensiones del INTAI.

\begin{tabular}{|c|c|c|}
\hline & \multicolumn{2}{|c|}{ Puntaje Promedio (1-100) } \\
\hline Transparencia Activa & Transparencia Pasiva & Institucional \\
\hline 80.96 & 79.32 & 53.68 \\
\hline
\end{tabular}
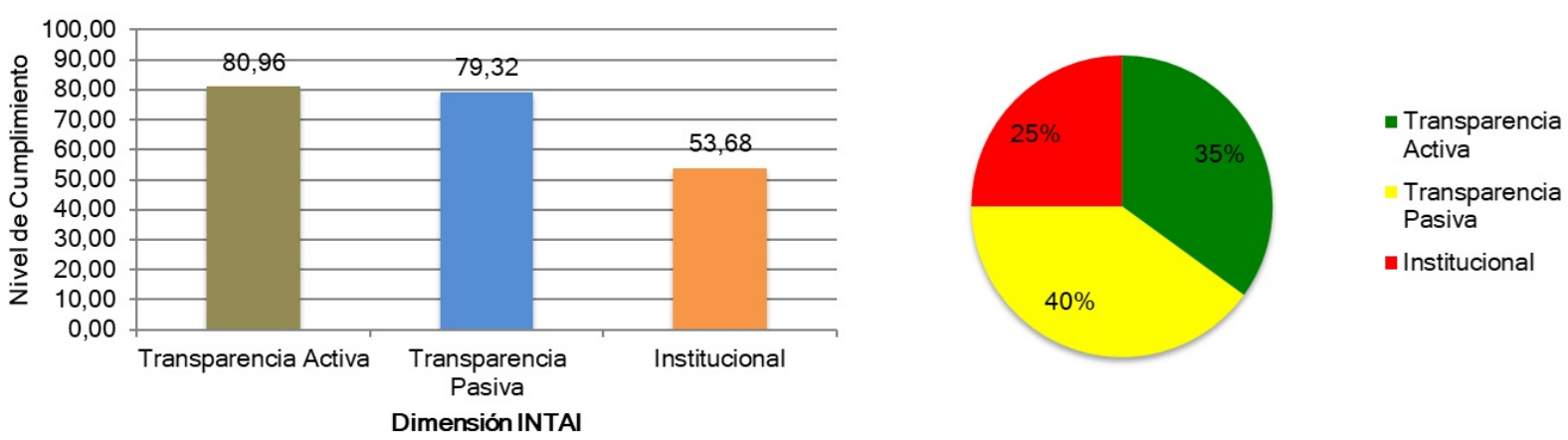

Fuente: Uruguay. Mirador de Gobierno Abierto (2021).

En particular, los resultados de la dimensión institucional - que se pueden ver en la tabla 5- son los que obtienen los menores puntajes en comparación con las demás dimensiones del índice, y son los resultados de gestión documental y de archivos los que tienen una puntuación general por debajo del promedio.

Del informe producido por la UAIP, se destaca que la subdimensión Gestión documental y de archivos es la "más rezagada del estudio, y con resultados dispares en cada uno de los aspectos evaluados" (UAIP, 2020).

A partir del análisis realizado se distingue como cumplimiento aceptable la existencia de fondos documentales en las organizaciones en el $76 \%$ de los sujetos obligados y un espacio digital acondicionado para el funcionamiento de los archivos digitales con el mismo porcentaje de cumplimiento. Mientras que, la provisión de espacios físicos preparados para el funcionamiento de los archivos con un $72 \%$ de cumplimiento entre los sujetos obligados; al igual que la existencia de sistemas de seguridad de los documentos en soporte digital.

Sin embargo, el grado de cumplimiento baja sustantivamente en lo referente a la posesión de sistemas de seguridad de los documentos en soporte físico (analógico, papel), con únicamente un $48 \%$ de cumplimiento entre los sujetos obligados y solamente un $40 \%$ en la normalización de los procesos de reproducción de los documentos y de constitución de las comisiones de evaluación documental institucional.

Este porcentaje baja aún más al momento de dar cumplimiento a la obligación de profesionalización de los servicios, únicamente cumplido por el $32 \%$ de los sujetos obligados, al igual que el deber de identificación de series documentales y de elaboración de las tablas de plazo precaucional de documentos, que también es acatado por el $32 \%$ de los organismos. 
TABLA 5

Resultados de la dimensión institucional, subdimensión Gestión de documentos y archivos.

\begin{tabular}{|l|c|c|c|}
\hline Preguntas subdimensión Gestión documental y de archivos & sí & Parcialmente & NO \\
\hline $\begin{array}{l}\text { GDA1: ¿Existe fondo(s) documental(es) y/o colecciones } \\
\text { identificado(s) y organizado(s) archivísticamente, cualquiera sea } \\
\text { el soporte en donde esté contenida la información? }\end{array}$ & 17 & 2 & 6 \\
\hline $\begin{array}{l}\text { GDA2: ¿Existen profesionales archivólogos en los Archivos } \\
\text { Administrativos e Históricos, on las Unidades de Gestión } \\
\text { Documental? }\end{array}$ & 8 & 0 & 17 \\
\hline $\begin{array}{l}\text { GDA3: ¿Existen espacios físicos acondicionados para el } \\
\text { funcionamiento de los Archivos; con la logística e infraestructura } \\
\text { necesaria para desarrollar los procesos archivísticos? }\end{array}$ & 14 & 4 & 7 \\
\hline $\begin{array}{l}\text { GDA4: ¿Existe un espacio digital acondicionado para el } \\
\text { funcionamiento de los Archivos digitales, logistica e } \\
\text { infraestructura necesaria para los procesos archivísticos? }\end{array}$ & 15 & 4 & 6 \\
\hline $\begin{array}{l}\text { GDA5: ¿Existen Sistemas de seguridad de los documentos en } \\
\text { soporte físico (analógico, papel) junto con una gestión de los } \\
\text { riesgos definidae implementada? }\end{array}$ & 8 & 4 & 13 \\
\hline $\begin{array}{l}\text { GDA6: ¿Existen Sistemas de seguridad de Ios documentos en } \\
\text { soporte digital (respaldo, acceso, confidencialidad, autenticidad y } \\
\text { trazabilidad) junto con gestión de los riesgos definida e } \\
\text { implementada? }\end{array}$ & 12 & 6 & 7 \\
\hline $\begin{array}{l}\text { GDA7: ¿Están normalizados los procesos de reproducción de los } \\
\text { documentos (protocolos de microfilmación y de digitalización) y } \\
\text { definidos los sistemas de preservación digital? }\end{array}$ & 4 & 6 & 15 \\
\hline $\begin{array}{l}\text { GDA8: ¿Está constituida la Comisión de evaluación Documental } \\
\text { institucional establecida en la normativa? }\end{array}$ & 6 & 4 & 15 \\
\hline $\begin{array}{l}\text { GDA9: ¿Están identificadas las series documentales y elaboradas } \\
\text { las Tablas de Plazos Precaucionales de documentos? }\end{array}$ & 4 & 4 & 17 \\
\hline
\end{tabular}

Fuente: Uruguay. Mirador de Gobierno Abierto (2021).

\section{Conclusiones}

Si bien se trata de una única pequeña muestra, los resultados de la aplicación del INTAI en los organismos que participaron como pilotos del proyecto evidencian que aún falta mucho trabajo por hacer en materia de gestión de documentos y archivos en la administración pública uruguaya.

En el país se cuenta con un marco legal y normas técnicas más que suficientes para cumplir con los procesos y los procedimientos técnicos, tales como la identificación, la clasificación, la descripción, la evaluación, la transferencia y la eliminación de documentos. Todos ellos representan herramientas de gestión documental fundamentales al momento de minimizar los riesgos que significa la pérdida de información o la falta de eficiencia y eficacia en la gestión de esta y como resultado la imposibilidad del ejercicio del derecho de acceso a la información pública y la transparencia.

La aprobación del Modelo de Gestión Documental de la RTA por parte de Uruguay y su implementación a nivel nacional a través de las guías técnico-metodológicas de gestión documental de la UAIP representan una oportunidad para seguir un ejemplo confiable, basado en estándares y buenas prácticas internacionales, que sin dudas permitirá obtener buenos resultados. 
Es necesario tener en cuenta que, para el caso de Uruguay existe coincidencia entre los sujetos obligados de la Ley del Sistema Nacional de Archivos No 18.220 de 20 de diciembre de 2007 y los de la Ley de Derecho de acceso a la Información pública, $N^{\circ} 18.381$ de 17 de octubre de 2008, a saber: órganos y organismos públicos, estatales o no estatales. Por lo tanto, no existe impedimento jurídico para la implementación de las guías técnico-metodológicas de gestión documental, que además, recogen la normativa mencionada anteriormente.

La implementación de las guías técnico-metodológicas y de la normativa nacional mencionada requiere de la planificación de recursos para garantizar, como dice la Ley 18.220, "las condiciones necesarias, en cuanto a edificios y equipamiento, de acuerdo a especificaciones técnicas". De la misma forma, la profesionalización de las áreas de gestión documental y archivos de las organizaciones también demanda en sus plantillas de funcionarios la incorporación de licenciados en archivología. Sin embargo, es necesario considerar que ambas normas, tienen más de diez años desde su promulgación y reglamentación, por lo que las previsiones de financiamiento y recursos ya deberían haberse contemplado.

Por su parte, algunos de los plazos previstos en las normas y recogidos en las guías ya se han completado. Como el de un año para la remisión de las tablas de plazos precaucionales de documentos a la Comisión de Evaluación Documental de la Nación del Archivo General de la Nación; o el plazo de cuatro años, para adecuar los archivos de los sujetos obligados, durante el cual "no serán pasibles de sanción en caso de denegación de acceso fundada en la imposibilidad de ubicar la información.”, Por lo que, su inobservancia debería ser controlada por el órgano rector de las políticas archivísticas nacionales.

Implementar las guías es, en definitiva, dar cumplimiento a normativa nacional preceptiva para esos órganos y organismos públicos, estatales y no estatales. Es necesario que todos estos instrumentos legislativos, normativos y metodológicos sean vistos de forma integrada con la implementación de una política nacional archivística. Esto es, una directriz que guíe las acciones en materia de gestión de documentos y archivos y que represente la voluntad del país sobre el rumbo a seguir en la materia.

\section{ReFERENCIAS}

AGESIC. Agencia de Gobierno Electrónico y Sociedad de la Información y del Conocimiento. (2018). Plan de Acción Nacional de Gobierno Abierto 2018-2020. Recuperado de https://www.gub.uy/agencia-gobierno-electronico-sociedad-informacion-conocimiento/sites/agencia-gobi erno-electronico-sociedad-informacion-conocimiento/files/2019-02/4to_plan_accion_gobierno_abierto_vf_ 26_11_2018_0.pdf

CLAD. Centro Latinoamericano de Administración para el Desarrollo. (2016). Carta Iberoamericana de Gobierno Abierto, 25-56. Recuperado de https://clad.org/wp-content/uploads/2020/10/0-Cartas-iberoamericanas-CLA D-2019.pdf

Delpiazzo, C. (2003). De la publicidad a la transparencia en la gestión administrativa. Revista de Derecho de la Universidad de Montevideo, 3, 113-124. Recuperado de http://revistaderecho.um.edu.uy/wp-content/uploads/ 2012/12/Delpiazzo-De-la-publicidad-a-la-transparencia-en-la-gestion-administrativa.pdf

Delpiazzo, C. (2013). Afirmación y evolución del principio de juridicidad. En J. O. Santofimio, H. Santaella y A. Matilla (coordinadores), Ensayos de derecho público en memoria de Maurice Hauriou (pp. 197-216). Bogotá: Universidad Externado de Colombia.

Francia. Asamblea Nacional. (1789). Declaración de los Derechos del Hombre y del Ciudadano. Recuperado de https:/ /www.conseil-constitutionnel.fr/sites/default/files/as/root/bank_mm/espagnol/es_ddhc.pdf

Lavalle Cobo, D. (2009). Derecho de acceso a la información pública. Buenos Aires: Astrea.

Mendel, T. (2009). El derecho a la información en América Latina: comparación jurídica. Quito: Unesco. Recuperado de http://unesdoc.unesco.org/images/0018/001832/183273s.pdf. 
Muñoz Lorente, J. (1999). Libertad de información y derecho al honor en el Código Penal de 1995. Valencia: Tirant lo Blanch.

OEA. Organización de los Estados Americanos. (1969, noviembre 7-22). Conferencia Especializada Interamericana de Derechos Humanos. Convención Americana sobre Derechos Humanos (Pacto de San José de Costa Rica). Washington DC: OEA-Secretaría General. Recuperado de https://www.oas.org/es/cidh/mandato/basicos/act as-conferencia-interamericana-derechos-humanos-1969.pdf

OEA. Organización de los Estados Americanos. (2010). Ley Modelo de Acceso a la Información Administrativa. Recuperado de http://www.oas.org/juridico/english/ley_modelo_acceso.pdf.

OEA. Organización de los Estados Americanos (2010). Comentarios y guia de implementación para la Ley Modelo Interamericana sobre Acceso a la Información. OEA/Set./ CP/CAJP-2841/10. 23 abril 2010. Recuperado de ht tp://www.oas.org/es/sla/ddi/docs/AG-RES_2841_XL-O-10_esp.pdf

OMS. Organización Mundial de la Salud. (2015). Nota descriptiva 323. Recuperado de https://www.who.int/es/new s-room/fact-sheets/detail/human-rights-and-health.

ONU. Organización de las Naciones Unidas. (1948, diciembre 10). Declaración Universal de los Derechos Humanos. Recuperado de https://www.un.org/es/universal-declaration-human-rights/.

PNUD. Programa de las Naciones Unidas para el Desarrollo. (2015). Objetivos de Desarrollo Sostenible. Objetivo 7: Energía asequible y no contaminante. Recuperado de https://www.undp.org/content/undp/es/home/sustainab le-development-goals/goal-7-affordable-and-clean-energy.html.

RAE. Real Academia Española (2014). Acceso. En Diccionario de la lengua española. (23a . ed.). Recuperado de http s://dle.rae.es.

Ramos, L. y Villar, A. (2013). El acceso a la información pública y los archivos en Uruguay. Palabra Clave, 3(1), 45-51. Recuperado de: http://www.memoria.fahce.unlp.edu.ar/art_revistas/pr.5819/pr.5819.pdf

Rotondo Tornaría, F. (1982). Aproximación a la participación del administrado a la luz de los principios generales. Revista de la Facultad de Derecho y Ciencias Sociales, 26(1), 53-69.

RTA. Red de Transparencia y Acceso a la Información (2014). Modelo de Gestión Documental de la Red de Transparencia y Acceso a la Información. Recuperado de http://mgd.redrta.org/mgd/site/edic/base/port/inicio .htm

Torres, N. (2014). Hacia una política integral de gestión de la información pública: todo lo que siempre quisimos saber sobre archivos. Buenos Aires: Universidad de Palermo.

Transparencia Internacional. (2020). Índice de percepción de la corrupción. Recuperado de https://images.transparen cycdn.org/images/2020_Report_CPI_EN.pdf

UAIP. Unidad de Acceso a la Información Pública. (2019a). Cuestionario web INTAI. Recuperado de https://www.gub.uy/unidad-acceso-informacion-publica/comunicacion/noticias/cuestionario-web-permit e-organismos-evaluar-aspectos-su-nivel-transparencia

UAIP. Unidad de Acceso a la Información Pública (2019b). Metodología del Índice Nacional de Transparencia y Acceso a la Información. Recuperado de https://www.gub.uy/unidad-acceso-informacion-publica/comunicacion/publ icaciones/metodologia-del-indice-nacional-transparencia-acceso-informacion-intai

UAIP. Unidad de Acceso a la Información Pública (2020). Guías técnicometodológicas de gestión documental y administración de archivos. Recuperado de https://www.gub.uy/unidad-acceso-informacion-publica/sites/unidad-acceso-informacion-publica/files/do cumentos/publicaciones/guia\%20tecnica\%20gestion\%20documental\%20uaip\%20-\%20version\%20digital.pdf

Uruguay. Mirador del Gobierno Abierto. Recuperado de https://miradordegobiernoabierto.agesic.gub.uy/SigesVisu alizador/ga/o/GA

Uruguay. Poder Judicial (1999, octubre 13). Sentencia 253 del 13 de octubre de 1999. (Sentencia N²53/1999). Recurso de casación.

Uruguay. Poder Judicial (2013, marzo 13) Sentencia 185 del 13 de marzo de 2013. (Sentencia Nº 185/2013) Proceso de inconstitucionalidad. 
Uruguay. Poder Judicial (2018, junio 4). Sentencia 802 del 4 de junio de 2018. (Sentencia No 802/2018). Recurso de casación.

Uruguay. Poder Judicial. Juzgado Letrado de Primera Instancia de lo Contencioso Administrativo de 1er. Turno (2011, octubre 21). Sentencia 144 de 21 de octubre de 2011. (Sentencia No 144/2011).

Uruguay. Poder Judicial. Tribunal de Apelaciones en lo Civil de 1er. Turno (2013, noviembre 6). Sentencia 204 del 6 de noviembre de 2013. (Sentencia $N^{\circ}$ 204/2013) Proceso de inconstitucionalidad.

Uruguay. Poder Judicial. Tribunal de Apelaciones en lo Civil de $6^{\circ}$ turno (2017, septiembre 5). Sentencia 104 del 5 de septiembre de 2017. (Sentencia No 104/2017). Proceso civil extraordinario.

Uruguay. Poder Judicial. Tribunal de Apelaciones en lo Civil de 1er. Turno (2019, mayo 15) Sentencia 74 del 15 de mayo de 2019. (Sentencia $\mathrm{N}^{\circ} 74 / 2019$ ). Proceso civil extraordinario.

Uruguay. (2007, diciembre 20). Ley $N^{\circ} 18.220$ Sistema Nacional de Archivos: conservación y organización del patrimonio documental de la nación. Montevideo: IMPO - Centro Oficial de Información. Recuperado de https://www.im po.com.uy/bases/leyes/18220-2007_

Uruguay. (2008, octubre 17). Ley $N^{\circ} 18.381$ Ley sobre derecho de acceso a la información pública. Montevideo: IMPOCentro oficial de información.

Uruguay. (2012, octubre 31). Decreto $\mathrm{N}^{\circ}$ 355/2012 Reglamentación de la regulación de la actividad archivística, creando el Sistema Nacional de Archivos. Recuperado de https://www.impo.com.uy/bases/decretos/355-201 $2 / 14$

Uruguay. Poder Judicial. (2014, abril 24). Ministro Pérez Manrique sostuvo que la ley de acceso a la información pública salvó su primer test de constitucionalidad. Recuperado de https://www.poderjudicial.gub.uy/novedades/noticias-judiciales/item/535-ministro-perez-manrique-sostu vo-que-la-ley-de-acceso-a-la-informacion-publica-salvo-su-primer-test-de-constitucionalidad.html

Villar, A.; Ramos, L.; Piccini, J. y Hernández, F. (2020). Construcción de políticas archivísticas en el Uruguay: consideraciones teóricas y la aplicación de métodos cuantitativos en la gestión documental y archivos. Boletín de la $A N A B A D, 70(2), 97-117$.

\section{Notas}

1. Proyecto de Código de Práctica sobre la Gestión Documental de Reino Unido, citado en los Comentarios y guía de implementación para la Ley Modelo Interamericana sobre Acceso a la Información.

2. Recuperado de https://www.gub.uy/unidad-acceso-informacion-publica/comunicacion/noticias/cuestionario-webpermite-organismos-evaluar-aspectos-su-nivel-transparencia

3. Obligación también incluida en el artículo 7 de la Ley 19.768, sobre la regulación del ejercicio de la profesión universitaria de los archivólogos. Ver: https://www.impo.com.uy/bases/leyes/19768-2019/7.

4. Obligación establecida en el artículo 8 de la Ley 19.768. Recuperado de https://www.impo.com.uy/bases/ leyes/19768-2019/8

5 Literal agregado por el Decreto 70/015, del 3 de febrero de 2015, artículo 1.

6 Conforme a lo dispuesto en el artículo 1 del Decreto 428/999, del 30 de diciembre de 1999. 